\title{
mRNA vaccines take on immune tolerance
}

\author{
A tolerizing mRNA vaccine prevents autoimmune disease in mice.
}

\section{Christine M. Wardell and Megan K. Levings}

V accines based on mRNA have dominated the headlines during the COVID-19 pandemic owing to their high efficacy and unprecedented speed of development and manufacture. In a recent paper in Science, Ugur Sahin, who led the development of a COVID19 mRNA vaccine ${ }^{1}$, and coauthors have now harnessed this vaccine technology to suppress, rather than prime, antigen-specific immune responses ${ }^{2}$. Working with mouse models of multiple sclerosis, they show that immunization with a modified mRNA encoding a self antigen and delivered in a non-inflammatory lipoplex carrier leads to a dampening of autoimmunity through the activation of antigen-specific regulatory T cells (Fig. 1). Although further research is needed to assess the clinical potential of the strategy, a successful tolerizing vaccination approach could transform the treatment of autoimmune diseases, allergies and allogeneic transplantation.

mRNA vaccines consist of antigenencoding RNA encapsulated in lipid nanoparticles ${ }^{3}$. Sahin and colleagues initially developed mRNA vaccines with the aim of stimulating immunity against tumors ${ }^{4}$. In those studies, the net negative charge of the mRNA-lipid nanoparticle resulted in their macropinocytosis by splenic dendritic cells, activation of Toll-like receptor 7 (TLR7), production of interferon- $\alpha$, and triggering of antigen-specific immunity ${ }^{4}$. In parallel, Kariko and colleagues developed a modification of mRNA-based vaccines that incorporated 1-methylpseudouridine $(\mathrm{m} 1 \Psi)$, a naturally occurring component of eukaryotic $18 \mathrm{~S}$ ribosomal RNA, in place of uridine, finding that $\mathrm{m} 1 \Psi$ mRNA had a higher translational capacity and lower innate immune activation because it did not stimulate TLR7 $7^{5,6}$.

Reasoning that an anti-inflammatory, m1 1 -based mRNA vaccine could be advantageous for disease contexts where suppression of immunity was desired, in the new study Sahin and colleagues ${ }^{2}$ investigated whether $\mathrm{m} 1 \Psi$ mRNA vaccination could prevent autoimmunity. The authors focused on a mouse model of multiple sclerosis, experimental autoimmune

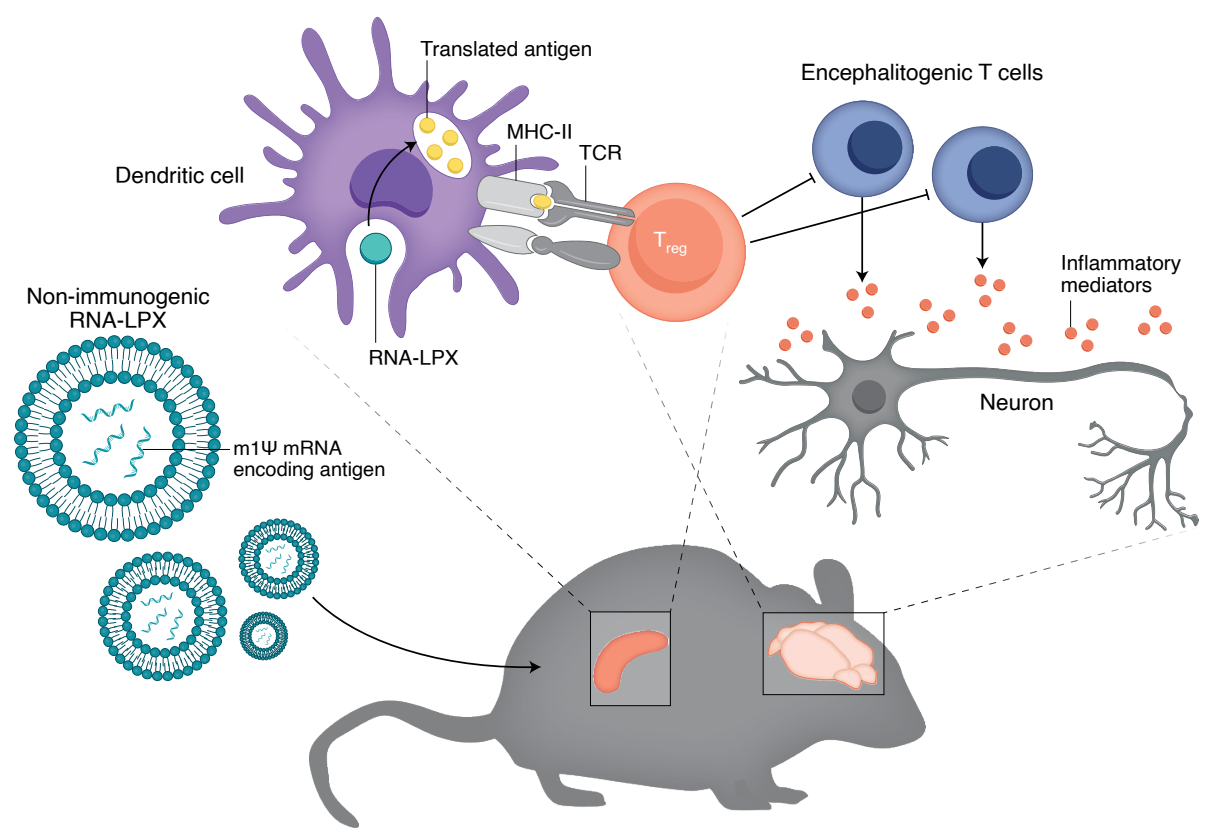

Fig. 1 | Inducing tolerance with an anti-inflammatory mRNA vaccine. Anti-inflammatory lipid nanoparticles containing myelin oligodendrocyte glycoprotein (MOG) $m 1 \Psi$ mRNA are specifically taken up by splenic dendritic cells following intravenous injection in mice. MOG m1Y mRNA is non-immunogenic and triggers the proliferation of $\mathrm{T}_{\text {reg }}$ cells that mediate antigen-specific and bystander suppression, preventing further myelin damage and paralysis. $m 1 \Psi, 1$-methylpseudouridine; MHC-II, major histocompatibility complex class II; RNA-LPX, RNA lipoplex; TCR, T cell receptor; $\mathrm{T}_{\text {reg' }}$ regulatory $\mathrm{T}$ cells.

encephalomyelitis (EAE), that can be triggered by immunization with a myelin oligodendrocyte glycoprotein peptide $\left(\mathrm{MOG}_{35-55}\right)$ and strong adjuvant. MOG is an autoantigen associated with multiple sclerosis; in EAE, MOG-specific T cells drive disease, causing progressive nerve damage. In the absence of adjuvant, administration of MOG m1 $\Psi$ mRNA in healthy mice induced significantly higher MOG-specific T cell proliferation than did administration of MOG uridine mRNA, but the m1 $1 \Psi$ mRNA-stimulated T cells expressed high levels of exhaustion markers and minimal amounts of inflammatory cytokines. Importantly, the m1 $\Psi$ mRNA formulation also stimulated proliferation of suppressive, MOG-specific FoxP3 ${ }^{+}$ regulatory $\mathrm{T}\left(\mathrm{T}_{\text {reg }}\right)$ cells.
After mice are immunized with MOG peptide and an adjuvant, clinical signs of disease appear in 10-15 days. To test whether m1 $1 \Psi$ mRNA could prevent disease, the authors first injected the MOG peptide and adjuvant and then administered $\mathrm{m} 1 \Psi$ mRNA encoding MOG or an irrelevant peptide 7 and 10 days later, before symptoms developed. Mice that received MOG m $1 \Psi$ mRNA were indeed protected from developing EAE, whereas mice treated with m $1 \Psi$ mRNA encoding an irrelevant peptide developed disease similarly to saline-treated mice. When the tolerizing treatment was delayed to the time of early disease onset (at around day 10, when partial paralysis is detected), only MOG m1 $\Psi$ mRNA prevented disease progression. Similar results were found in a relapsing-remitting 
EAE model induced by a proteolipid protein $\left(\mathrm{PLP}_{139-151}\right)$ peptide in SJL mice.

Analysis of the cellular immune response in MOG $\mathrm{m} 1 \Psi$ mRNA-treated mice revealed less $T$ cell infiltration and reduced demyelination in the brain and spinal cord. Furthermore, the spleens of protected mice contained MOG-specific T cells expressing low levels of activation markers, high levels of co-inhibitory molecules, and higher numbers of activated $\mathrm{T}_{\text {reg }}$ cells. The efficacy of the MOG m1 $\Psi$ mRNA treatment was significantly reduced by antibodies blocking CTLA-4 or PD-1, possibly as a result of interference with mechanisms of action in $\mathrm{T}_{\text {reg }}$ cells or by enhancement of $\mathrm{T}$ cell effector functions, respectively. Taken together, these data suggest that although m1 $\Psi$ mRNA vaccination promotes the expansion of $\mathrm{T}_{\text {reg }}$ cells, it does not delete pre-existing autoreactive T cells. Notably, m1 $\Psi$ mRNA administration did not induce MOG-specific autoantibodies, which is a potential risk of therapies involving administration of disease-relevant autoantigens.

Because multiple sclerosis is driven by several autoantigens, successful immune tolerizing interventions in patients might require $\mathrm{T}_{\text {reg }}$ cells that not only suppress cognate autoreactive $\mathrm{T}$ cells but also mediate bystander suppression of autoreactive T cells bearing other antigen specificities. To test whether m1 1 mRNA-expanded $\mathrm{T}_{\text {reg }}$ cells were capable of bystander suppression, the authors induced EAE with the PLP peptide and then treated them with MOG m1 $1 \Psi$ mRNA or PLP $\mathrm{m} 1 \Psi$ mRNA; both treatments led to similar reductions in symptoms, suggesting that $\mathrm{T}_{\text {reg }}$ cells activated by MOG $\mathrm{m} 1 \Psi$ mRNA were capable of bystander suppression. Moreover, in an EAE model triggered with five autoantigens, vaccination with m1 $\Psi$ mRNA encoding a single peptide or a mixture of four autoantigens provided protection, with a trend toward better protection with the latter strategy. Thus, more work is required to understand the role of antigen-specific versus bystander mechanisms of tolerance induction.

A potential safety concern of $\mathrm{m} 1 \Psi$ mRNA vaccination is the possibility of generalized immune suppression caused by long-lasting tolerogenic effects on the dendritic cells that take up RNA lipoplexes. To address this, Sahin and colleagues showed that prior treatment with $\mathrm{m} 1 \Psi$ mRNA did not prevent dendritic cells from upregulating activation markers and producing interferon- $\alpha$ when subsequently challenged with uridine mRNA in vivo, suggesting that the dendritic cells remain fully functional after m $1 \Psi$ internalization. Moreover, treatment with MOG m1 $\Psi$ mRNA did not hamper the development of $\mathrm{T}$ cell immunity to model antigens delivered by uridine mRNA vaccination. Thus, dendritic cells exposed to $1 \Psi$ mRNA are not refractory to activation, indicating that the anti-inflammatory effect of $\mathrm{m} 1 \Psi$ mRNA vaccination is remarkably specific.

Sahin and colleagues have clearly demonstrated the potential of RNA lipoplex vaccines to deliver a non-inflammatory form of an mRNA vaccine encoding a self antigen to prevent and limit autoimmune disease in mice. It is noteworthy that $\mathrm{m} 1 \Psi$-containing mRNA is also used for the COVID-19 mRNA vaccine ${ }^{1,7}$, indicating that the pro- versus anti-inflammatory nature of $\mathrm{m} 1 \Psi$ mRNA vaccines can be modulated depending on the specific antigen and specific encapsulating lipid formulation. In the case of the BNT162b2 vaccine for COVID-19, the antigen is a foreign protein formulated in an immunostimulatory lipid nanoparticle ${ }^{8}$. In the present study, the antigen is a self protein delivered in a non-immunogenic lipoplex formulation, and an extra mRNA purification step removes any residual immunostimulatory molecules ${ }^{2}$. This method allows antigen presentation in the absence of inflammation and co-stimulation, preferential expansion of pre-existing $\mathrm{T}_{\text {reg }}$ cells, and possibly also their de novo development.

The notion that vaccines based on nucleic acids could be effective in resetting immune responses in multiple sclerosis is not new: 15 years ago, vaccination with DNA encoding myelin basic protein was found to induce favorable trends on brain magnetic resonance imaging and beneficial antigen-specific immune effects ${ }^{9}$. This is also not the first time that the delivery of autoantigens in an anti-inflammatory format has been explored as a method to induce tolerance. Previous approaches have included peptide or protein delivery in anti-inflammatory formats using methods such as oral, nasal or subcutaneous administration, or delivery on carriers such as cells or major histocompatibility complex molecules ${ }^{10}$. However, the success of these DNA- and protein-based concepts in the clinic has been mixed, with generally underwhelming results so far $^{9,10}$. The major advantages of the RNA lipoplex antigen delivery system are that it is easily adaptable to any protein antigen (or indeed combination of antigens) and feasible and cost-effective at large scales ${ }^{3}$. This means the approach would be applicable in large numbers of people, and might also include repeat dosing.
Before $\mathrm{m} 1 \Psi$ mRNA vaccination to treat autoimmune diseases can be translated to humans, key questions related to defining optimal disease-specific autoantigen targets and to the longevity and specificity of the effect must be answered. By design, animal models of EAE are typically driven by one predominant, disease-causing autoantigen, whereas multiple sclerosis is driven by a complex polyclonal T cell repertoire ${ }^{10,11}$. More work will be needed to fully understand the capacity for, and breadth of, bystander suppression. The current report did not include formal demonstration of tolerance by a later rechallenge with the autoantigen in a non-tolerogenic format, or by the adoptive transfer of $\mathrm{T}_{\text {reg }}$ cells to naive mice (for demonstrating 'dominant' tolerance). Autoimmune diseases in humans are thought to involve continuous exposure to autoantigens, possibly coincident with inflammatory stimuli; therefore, it will be important to establish the length of the immunoregulatory effect and whether repeat vaccination is safe or advantageous. Finally, the fact that similar approaches can be used to stimulate immune responses ${ }^{1}$ underlines the need to fully understand the mechanisms that control the pro-versus anti-inflammatory nature of mRNA vaccines to eliminate the risk of deleterious immune stimulation. Nevertheless, the simplicity and remarkable potential of $\mathrm{m} 1 \Psi$ mRNA demonstrated by Sahin and colleagues is very encouraging.

\section{Christine M. Wardell(D) and Megan K. Levings (D) \\ BC Children's Hospital Research Institute and Department of Surgery, University of British Columbia, Vancouver, British Columbia, Canada. \\ $凶_{e-\text { mail: mlevings@bcchr.ca }}$}

Published online: 30 March 2021 https://doi.org/10.1038/s41587-021-00880-0

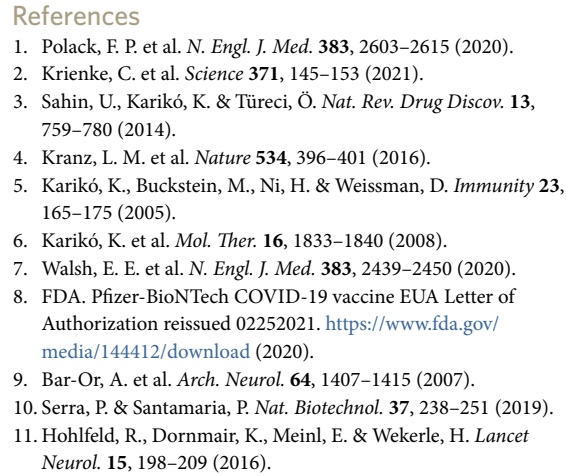

Competing interests

The authors declare no competing interests. 


\section{RESEARCH HIGHLIGHTS}

\section{Papers from the literature}

Selected by the Nature Biotechnology editors.

Multimodal pooled Perturb-CITE-seq screens in patient models define mechanisms of cancer immune evasion

Frangieh, C. J. et al. Nat. Genet. https://doi.org/10.1038/s41588-021-00779-1 (2021).

Enhancing grain-yield-related traits by CRISPR-Cas9 promoter editing of maize CLE genes

Liu, L. et al. Nat. Plants https://doi.org/10.1038/s41477-021-00858-5 (2021).

Global analysis of shared $\mathrm{T}$ cell specificities in human non-small cell lung cancer enables HLA inference and antigen discovery

Chiou, S.-H. et al. Immunity https://doi.org/10.1016/j.immuni.2021.02.014 (2021).

Massively parallel assessment of human variants with base editor screens

Hanna, R. E. et al. Cell https://doi.org/10.1016/j.cell.2021.01.012 (2021).

Ex utero mouse embryogenesis from pre-gastrulation to late organogenesis

Aguilera-Castrejon, A. et al. Nature https://doi.org/10.1038/s41586-021-03416-3 (2021).

Blastocyst-like structures generated from human pluripotent stem cells Yu, L. et al. Nature https://doi.org/10.1038/s41586-021-03356-y (2021).

Modelling human blastocysts by reprogramming fibroblasts into iBlastoids

Liu, X. et al. Nature https://doi.org/10.1038/s41586-021-03372-y (2021).

Follow us on Twitter @NatureBiotech \#nbtHighlight 